\title{
Existence and Ulam stability results of a coupled system for terminal value problems involving $\psi$-Hilfer fractional operator
}

\author{
Mohammed S. Abdo ${ }^{1,2^{*}}$ (D, Kamal Shah ${ }^{3}$, Satish K. Panchal ${ }^{1}$ and Hanan A. Wahash ${ }^{1}$
}

\section{"Correspondence:}

msabdo1977@gmail.com

'Department of Mathematics, Dr.

Babasaheb Ambedkar Marathwada

University, Aurangabad, India

${ }^{2}$ Department of Mathematics,

Hodeidah University, AL-Hodeidah, Yemen

Full list of author information is available at the end of the article

\begin{abstract}
The work reported in this paper deals with the study of a coupled system for fractional terminal value problems involving $\psi$-Hilfer fractional derivative. The existence and uniqueness theorems to the problem at hand are investigated. Besides, the stability analysis in the Ulam-Hyers sense of a given system is studied. Our discussion is based upon known fixed point theorems of Banach and Krasnoselskii. Examples are also provided to demonstrate the applicability of our results.
\end{abstract}

MSC: Primary 26A33; secondary 34A07; 93A30; 35R11

Keywords: Hilfer fractional differential equation; Terminal value problem; Existence and stability theory; Fixed point theorem

\section{Introduction}

Over the past few years, mathematicians have realized that fractional calculus has many applications in various scopes of applied science and engineering. Several researchers have employed the fractional calculus as an experiential style of describing the properties of natural phenomena such as chemistry, biology, physics, bioengineering, electrochemistry, finance, economic, etc., for more details, see [12, 20, 21, 23] and many other references. The interesting issue about this theme is that it is completely unlike classical derivatives, because it deals with arbitrary and noninteger order, e.g., the fractional derivative of noninteger order depends not just on the diagram of the function very near to the point but also on some chronicle.

Recently, there has been considerable growth in fractional differential equations (FDEs) involving several different fractional derivative operators, we indicate here the more famous operators like Riemann-Liouville (RL), Caputo, Hilfer, Hadamard, Katugampola, and several other generalized operators.

So, this implies that different categories of FDEs involving several fractional operators have been considered. Researchers who are concerned with this topic have presented many generalizations of fractional derivatives like Hilfer-Katugampola, HilferHadamard, $\psi$-Caputo, and $\psi$-Hilfer, see [10, 16-18, 24, 29]. Some existence, uniqueness,

(c) The Author(s) 2020. This article is licensed under a Creative Commons Attribution 4.0 International License, which permits use, sharing, adaptation, distribution and reproduction in any medium or format, as long as you give appropriate credit to the original author(s) and the source, provide a link to the Creative Commons licence, and indicate if changes were made. The images or other third party material in this article are included in the article's Creative Commons licence, unless indicated otherwise in a credit line to the material. If material is not included in the article's Creative Commons licence and your intended use is not permitted by statutory regulation or exceeds the permitted use, you will need to obtain permission directly from the copyright holder. To view a copy of this licence, visit http://creativecommons.org/licenses/by/4.0/. 
and Ulam-Hyers stability results of generalized FDEs have been studied, see the recent references $[1-4,9,11,22,25,30-34]$.

On the other hand, many interesting and recent results on the existence and stability of a coupled system for different categories of FDEs have been investigated, see the following studies $[6-8,26]$ and the references therein.

Terminal value problems (TVPs) for FDEs for the time being play a major role in the modeling of numerous phenomena in engineering, science, and simulation. In short, the existence results for ordinary and fractional TVPs have been studied by many investigators, see [5, 13-15, 27, 28, 35]. For example, Benchohra et al. in [13] obtained the existence and uniqueness of solution to the fractional implicit TVP

$$
{ }^{\rho} D_{a^{+}}^{\theta, \eta} y(t)=f\left(t, y(t),{ }^{\rho} D_{a^{+}}^{\theta, \eta} y(t)\right), \quad a<t \leq T, a>0,
$$

under the terminal condition

$$
y(T)=w \in \mathbb{R},
$$

where ${ }^{\rho} D_{a^{+}}^{\theta, \eta}$ is the fractional derivative of order $(\theta, \eta)$ in the Hilfer-Katugampola sense $(0<\theta<1,0 \leq \eta \leq 1), \rho>0$, and $f:(a, T] \times \mathbb{R} \rightarrow \mathbb{R}$ is a certain function.

Motivated by the aforementioned works, the target of this work is to investigate the existence, uniqueness, and Ulam-Hyers stability of solutions of a coupled system for fractional TVPs involving generalized Hilfer fractional derivative of the type

$$
\begin{cases}D_{a^{+}}^{\theta_{1}, \eta_{1} ; \psi} y(t)=f_{1}(t, x(t)), & a<t \leq T, a>0, \\ D_{a^{+}}^{\theta_{2}, \eta_{2} ; \psi} x(t)=f_{2}(t, y(t)), & a<t \leq T, a>0\end{cases}
$$

under the terminal conditions

$$
\left\{\begin{array}{l}
y(T)=w_{1} \in \mathbb{R} \\
x(T)=w_{2} \in \mathbb{R}
\end{array}\right.
$$

where $0<\theta_{i}<1,0 \leq \eta_{i} \leq 1, D_{a^{+}}^{\theta_{i}, \eta_{i}, \psi}(i=1,2)$ is the Hilfer fractional derivative of order $\theta_{i}$ and type $\eta_{i}$ with respect to $\psi$ and $f:(a, T] \times \mathbb{R} \rightarrow \mathbb{R}$ is a certain function under the conditions listed later.

As far as we know, no papers about a coupled system for fractional TVPs exist in the literature, specifically for those encompassing the generalized fractional derivative in the $\psi$-Hilfer sense. Moreover, the results of the problem at hand are obtained under minimal assumptions on nonlinear functions $f_{1}, f_{2}$.

The rest of the structure of this paper is as follows. In Sect. 2, we briefly state some essential definitions and the results that are applied throughout the paper. Section 3 studies the existence and uniqueness results on $\psi$-Hilfer FDEs with the terminal conditions via fixed point techniques of Banach and Krasnoselskii. The stability analysis in the concept Ulam-Hyers of the proposed system is investigated in Sect. 4. At the end, some examples are included to illustrate the applicability of the obtained results in Sect. 5. 


\section{Auxiliary results}

Let $[a, T] \subset \mathbb{R}^{+}$with $(0<a<T<\infty)$, we also consider $C[a, T]$ the Banach space of realvalued continuous functions defined on $[a, T]$ with the norm

$$
\|\sigma\|_{C}=\max _{t \in[a, T]}|\sigma(t)|, \quad \sigma \in C[a, T] .
$$

Let $\varsigma=\theta+\eta(1-\theta)$ where $0<\theta<1,0 \leq \eta \leq 1$, and let $\psi \in C^{1}[a, T]$ be an increasing function with $\psi^{\prime}(t) \neq 0$ for each $t \in[a, T]$. For $0<\varsigma \leq 1$, the weighted spaces $C_{1-\zeta ; \psi}[a, T]$, $C_{1-\zeta ; \psi}^{n}[a, T]$ of continuous functions are defined by [29]

$$
\begin{aligned}
& C_{1-\varsigma ; \psi}[a, T]=\left\{\sigma:(a, T] \rightarrow \mathbb{R} ;[\psi(t)-\psi(a)]^{1-\varsigma} \sigma(t) \in C[a, T]\right\}, \\
& C_{1-\zeta ; \psi}^{n}[a, T]=\left\{\sigma \in C^{n-1}[a, T], \sigma^{(n)} \in C_{1-\zeta ; \psi}[a, T]\right\} .
\end{aligned}
$$

Obviously, $C_{1-\varsigma ; \psi}[a, T]$ and $C_{1-5 ; \psi}^{n}[a, T]$ are Banach spaces endowed with the norms

$$
\begin{aligned}
& \|\sigma\|_{C_{1-\zeta ; \psi}}=\max _{t \in[a, T]}\left|[\psi(t)-\psi(a)]^{1-\varsigma} \sigma(t)\right|, \\
& \|\sigma\|_{C_{1-\zeta ; \psi}^{n}}=\sum_{j=0}^{n-1}\left\|\sigma^{(j)}\right\|_{C}+\left\|\sigma^{(n)}\right\|_{C_{1-\zeta ; \psi}},
\end{aligned}
$$

respectively. For $n=0, C_{1-\zeta ; \psi}^{0}[a, T]=C_{1-\zeta ; \psi}[a, T]$. Let us introduce the following space:

$$
E=\left\{\sigma(t): \sigma(t) \in C_{1-\zeta ; \psi}[a, T]\right\}
$$

endowed with the norm defined by

$$
\|\sigma\|_{E}=\|\sigma\|_{c_{1-\zeta ; \psi}} .
$$

It is easy to perceive that, for $\sigma \in E,\left(E,\|\sigma\|_{E}\right)$ is a Banach space. Then, for $(\sigma, \rho) \in E \times E$, the product space $\left(E \times E,\|(\sigma, \rho)\|_{E \times E}\right)$ is a Banach space too, where

$$
\|(\sigma, \rho)\|_{E \times E}=\max \left(\|\sigma\|_{E},\|\rho\|_{E}\right), \quad \sigma, \rho \in E .
$$

Definition 1 ([19, Sect. 2.5, Eq. (2.5.1)]) Let $\theta>0, \sigma \in L_{1}[a, T]$. Then the left-sided $\psi$-RL fractional integral of order $\theta$ of a function $\sigma$ w.r.t. $\psi$ is described by

$$
I_{a^{+}}^{\theta, \psi} \sigma(t)=\frac{1}{\Gamma(\theta)} \int_{a}^{t} \psi^{\prime}(s)(\psi(t)-\psi(s))^{\theta-1} \sigma(s) d s .
$$

Definition 2 ([19, Eq. (2.5.17)], [10, Definition 1]) Let $n-1<\theta<n$, and $\sigma \in C^{n}[a, T]$. Then the left-sided $\psi$-fractional derivatives in the concepts $\psi$-RL and $\psi$-Caputo of order $\theta$ of a function $\sigma$ w.r.t. $\psi$ are described by

$$
D_{a^{+}}^{\theta, \psi} \sigma(t)=D^{n, \psi} I_{a^{+}}^{n-\theta, \psi} \sigma(t)
$$


and

$$
{ }^{C} D_{a^{+}}^{\theta, \psi} \sigma(t)=I_{a^{+}}^{n-\theta, \psi} \sigma_{\psi}^{[n]}(t)
$$

respectively, where $\sigma_{\psi}^{[n]}(t)=D^{n, \psi} \sigma(t), D^{n, \psi}=\left[\frac{1}{\psi^{\prime}(t)} \frac{d}{d t}\right]^{n}$, and $n=[\theta]+1$.

Definition 3 ([29, Definition 7]) Let $\varsigma=\theta+\eta(n-\theta)$ where $n-1<\theta<n \in \mathbb{N}, 0 \leq \eta \leq 1$, and $\sigma \in C^{n}[a, T]$. Then the left-sided $\psi$-fractional derivative in the concept $\psi$-Hilfer of order $\theta$ and type $\eta$ of a function $\sigma$ w.r.t. $\psi$ is given by

$$
\begin{aligned}
D_{a^{+}}^{\theta, \eta, \psi} \sigma(t) & =I_{a^{+}}^{\eta(n-\theta) ; \psi} D^{n, \psi} I_{a^{+}}^{(1-\eta)(n-\theta) ; \psi} \sigma(t) \\
& =I_{a^{+}}^{\eta(n-\theta) ; \psi} D_{a^{+}}^{\zeta ; \psi} \sigma(t),
\end{aligned}
$$

where

$$
D_{a^{+}}^{\zeta ; \psi} \sigma(t)=D^{n, \psi} I_{a^{+}}^{(1-\eta)(n-\theta) ; \psi} \sigma(t) .
$$

Lemma 1 ([32, Lemma 1]) Let $\theta>0$ and $0<\varsigma \leq 1$. Then $\left(I_{a^{+}}^{\theta, \psi}\right.$ is bounded $): C_{1-\zeta ; \psi}[a, T] \rightarrow$ $C_{1-\zeta ; \psi}[a, T]$.

Lemma 2 ([32, Lemma 1], [29, Lemma 4]) Let $\theta>0,0<\varsigma \leq 1$, and $\sigma \in C_{1-\varsigma, \psi}[a, T]$. If $\varsigma<\theta$, then $\left(I_{a^{+}}^{\theta, \psi}\right.$ is bounded $): C_{1-\varsigma ; \psi}[a, T] \rightarrow C[a, T]$. Besides,

$$
I_{a^{+}}^{\theta ; \psi} \sigma(a)=\lim _{t \rightarrow a^{+}} I_{a^{+}}^{\theta ; \psi} \sigma(t)=0
$$

Definition 4 [32, Definition 3] The weighted continuous spaces $C_{1-\varsigma ; \psi}^{\theta, \eta}[a, T]$ and $C_{1-\varsigma ; \psi}^{\varsigma}[a, T]$ are described by

$$
C_{1-\varsigma ; \psi}^{\theta, \eta}[a, T]=\left\{\sigma \in C_{1-\varsigma ; \psi}[a, T], D_{a^{+}}^{\theta, \eta ; \psi} \sigma \in C_{1-\varsigma ; \psi}[a, T]\right\}
$$

and

$$
C_{1-\zeta ; \psi}^{\varsigma}[a, T]=\left\{\sigma \in C_{1-\varsigma ; \psi}[a, T], D_{a^{+}}^{\zeta \psi} \sigma \in C_{1-\varsigma ; \psi}[a, T]\right\}
$$

where $\varsigma=\theta+\eta(1-\theta), 0<\theta<1$, and $0 \leq \eta \leq 1$. Observe that

$$
C_{1-\zeta ; \psi}^{\varsigma}[a, T] \subset C_{1-\zeta ; \psi}^{\theta, \eta}[a, T] \subset C_{1-\zeta ; \psi}[a, T] \subset C[a, T] .
$$

Lemma 3 ([2, Lemma 2.3]) Let $\varsigma=\theta+\eta(1-\theta)$ where $0<\theta<1,0 \leq \eta \leq 1$, and $\sigma \in$ $C_{1-\zeta ; \psi}^{\varsigma}[a, T]$. Then

$$
I_{a^{+}}^{\varsigma ; \psi} D_{a^{+}}^{\varsigma ; \psi} \sigma=I_{a^{+}}^{\theta ; \psi} D_{a^{+}}^{\theta, \eta ; \psi} \sigma \quad \text { and } \quad D_{a^{+}}^{\zeta ; \psi} I_{a^{+}}^{\theta ; \psi} \sigma=D_{a^{+}}^{\eta(1-\theta) ; \psi} \sigma .
$$

Lemma 4 ([29, Theorem 7]) Let $\theta>0,0<\varsigma \leq 1$, and $\sigma \in C_{1-\varsigma}[a, T], \eta \in[0,1]$. Then

$$
D_{a^{+}}^{\theta, \psi} I_{a^{+}}^{\theta, \psi} \sigma(t)=\sigma(t) .
$$


Lemma 5 ([29, Theorem 1]) Let $\theta>0,0<\varsigma \leq 1$, and $\sigma \in C_{1-\varsigma ; \psi}[a, T]$ and $I_{a^{+}}^{1-\varsigma ; \psi} \sigma \in$ $C_{1-\varsigma, \psi}^{1}[a, T]$. Then

$$
I_{a^{+}}^{\varsigma ; \psi} D_{a^{+}}^{\varsigma, \psi} \sigma(t)=\sigma(t)-\frac{I_{a^{+}}^{1-\varsigma ; \psi} \sigma(a)}{\Gamma(\varsigma)}(\psi(t)-\psi(a))^{\varsigma-1}
$$

Lemma $6\left(\left[19\right.\right.$, Property 2.18]) Let $t>a$, and consider $\chi^{\varsigma}(t):=[\psi(t)-\psi(a)]^{\varsigma^{-1}}$. Then, for $\theta>0$ and $\varsigma>0$,

$$
I_{a^{+}}^{\theta, \psi} \chi^{\varsigma}(t)=\frac{\Gamma(\varsigma)}{\Gamma(\theta+\varsigma)}(\psi(t)-\psi(a))^{\theta+\varsigma-1} .
$$

Besides, for $0<\theta<1$,

$$
D_{a^{+}}^{\theta, \psi} \chi^{\theta}(t)=0
$$

Theorem 1 ([36, Theorem 1.45]) Let $\Xi$ be a Banach space, and let $\varnothing \subset \Xi$ be a nonempty, closed, convex, and bounded set, and $\Pi_{1}, \Pi_{2}$ be two operators satisfying

(i) $\Pi_{1} u+\Pi_{2} v \in \varnothing$ for all $u, v \in \varnothing$;

(ii) $\Pi_{1}$ is continuous and compact;

(iii) $\Pi_{2}$ is a contraction operator.

Then there exists $u^{*} \in \varnothing$ such that $u^{*}=\Pi_{1} u^{*}+\Pi_{2} u^{*}$.

Theorem 2 ([36, Theorem 1.41]) Let $\Xi$ be a Banach space and $\varnothing$ be a nonempty closed subset of $\Xi$, then any contraction operator $\Pi: \varnothing \rightarrow \varnothing$ has a unique fixed point.

The results we study in this paper will only concern $0<\theta<1(n=1)$.

\section{Existence and uniqueness results}

To shorten the length of equations, we set $\mathcal{K}_{\psi}^{\varsigma}(t, a):=[\psi(t)-\psi(a)]^{\varsigma^{-1}}$ and $\mathcal{H}_{\psi}^{\theta}(t, s):=$ $\psi^{\prime}(s)[\psi(t)-\psi(s)]^{\theta-1}$.

Theorem 3 Let $\varsigma=\theta+\eta(1-\theta)$ where $0<\theta<1$ and $0 \leq \eta \leq 1$. If $\sigma:(a, T] \rightarrow \mathbb{R}$ is $a$ function such that $\sigma(\cdot) \in C_{1-\varsigma, \psi}[a, T]$, then $y \in C_{1-\varsigma, \psi}^{\varsigma}(a, T]$ satisfies the TVP for $\psi$-Hilfer FDEs

$$
\begin{aligned}
& D_{a^{+}}^{\theta, \eta ; \psi} y(t)=\sigma(t), \quad t \in(a, T], a>0, \\
& y(T)=w \in \mathbb{R},
\end{aligned}
$$

if and only if $y$ fulfills the following fractional integral equation:

$$
y(t)=\frac{\mathcal{K}_{\psi}^{s}(t, a)}{\mathcal{K}_{\psi}^{s}(T, a)}\left[w-\frac{1}{\Gamma(\theta)} \int_{a}^{T} \mathcal{H}_{\psi}^{\theta}(T, s) \sigma(s) d s\right]+\frac{1}{\Gamma(\theta)} \int_{a}^{t} \mathcal{H}_{\psi}^{\theta}(t, s) \sigma(s) d s .
$$

Proof Assume that $y \in C_{1-\varsigma, \psi}^{\zeta}[a, T]$ is the solution of the TVP for $\psi$-Hilfer FDEs (7)-(8). According to the definitions of $C_{1-\varsigma, \psi}^{\varsigma}(a, T]$ and $D_{a^{+}}^{\varsigma ; \psi}$, applying Lemma 1 , we have

$$
I_{a^{+}}^{1-\varsigma, \psi} y(t) \in C_{1-\varsigma, \psi}[a, T] \quad \text { and } \quad D_{a^{+}}^{\varsigma ; \psi} y(t)=D^{1, \psi} I_{a^{+}}^{1-\varsigma, \psi} y(t) \in C_{1-\varsigma, \psi}[a, T] .
$$


Thanks to the definition of $C_{1-\varsigma, \psi}^{n}[a, T]$, we have

$$
I_{a^{+}}^{1-\varsigma, \psi} y(t) \in C_{1-\varsigma, \psi}^{1}[a, T]
$$

Take advantage of Lemma 5 to get

$$
I_{a^{+}}^{\varsigma ; \psi} D_{a^{+}}^{\varsigma, \psi} y(t)=y(t)-\frac{I_{a^{+}}^{1-\varsigma ; \psi} y(a)}{\Gamma(\varsigma)} \mathcal{K}_{\psi}^{\varsigma}(t, a), \quad t \in(a, T]
$$

It follows from the assumption $y \in C_{1-\varsigma, \psi}^{\zeta}[a, T]$, Lemma 3 , and equation (7) that

$$
I_{a^{+}}^{\zeta ; \psi} D_{a^{+}}^{\zeta ; \psi} y(t)=I_{a^{+}}^{\theta ; \psi} \sigma(t)
$$

Equating both sides of equations (12) and (13), we find that

$$
y(t)=\frac{I_{a^{+}}^{1-\varsigma ; \psi} y(a)}{\Gamma(\varsigma)} \mathcal{K}_{\psi}^{\varsigma}(t, a)+I_{a^{+}}^{\theta ; \psi} \sigma(t)
$$

Using the terminal condition $y(T)=w$, we get

$$
I_{a^{+}}^{1-\varsigma ; \psi} y(a)=\frac{\Gamma(\varsigma)}{\mathcal{K}_{\psi}^{\varsigma}(T, a)}\left[w_{1}-I_{a^{+}}^{\theta ; \psi} \sigma(T)\right]
$$

Now, from equations (14) and (15), we conclude that

$$
y(t)=\frac{\mathcal{K}_{\psi}^{s}(t, a)}{\mathcal{K}_{\psi}^{5}(T, a)}\left[w-\frac{1}{\Gamma(\theta)} \int_{a}^{T} \mathcal{H}_{\psi}^{\theta}(T, s) \sigma(s) d s\right]+\frac{1}{\Gamma(\theta)} \int_{a}^{t} \mathcal{H}_{\psi}^{\theta}(t, s) \sigma(s) d s
$$

Hence $y(t)$ satisfies the TVP for $\psi$-Hilfer FDEs (7)-(8).

Conversely, suppose that $y \in C_{1-\zeta, \psi}^{\zeta}[a, T]$ satisfying integral equation (9). Applying fractional derivative $D_{a^{+}}^{\varsigma ; \psi}$ on both sides of integral equation (9) and employing Lemmas 6 and 3 , we will surely find

$$
\begin{aligned}
D_{a^{+}}^{\varsigma ; \psi} y(t) & =\frac{1}{\mathcal{K}_{\psi}^{\zeta}(T, a)}\left[w-I_{a^{+}}^{\theta ; \psi} \sigma(T)\right] D_{a^{+}}^{\varsigma ; \psi} \mathcal{K}_{\psi}^{\zeta}(t, a)+D_{a^{+}}^{\varsigma ; \psi} I_{a^{+}}^{\theta ; \psi} \sigma(t) \\
& =D_{a^{+}}^{\eta(1-\theta) ; \psi} \sigma(t),
\end{aligned}
$$

where $D_{a^{+}}^{\varsigma ; \psi} \mathcal{K}_{\psi}^{\varsigma}(t, a)=0$. From (10), we have $D_{a^{+}}^{\varsigma ; \psi} y \in C_{1-\varsigma ; \psi}[a, T]$, then (16) implies

$$
D_{a^{+}}^{\varsigma ; \psi} y(t)=D^{1, \psi} I_{a^{+}}^{1-\eta(1-\theta), \psi} \sigma(t)=D_{a^{+}}^{\eta(1-\theta) ; \psi} \sigma(t) \in C_{1-\varsigma ; \psi}[a, T] .
$$

As $\sigma(t) \in C_{1-\varsigma ; \psi}[a, T]$, it follows from Lemma 1 that

$$
I_{a^{+}}^{1-\eta(1-\theta) ; \psi} \sigma \in C_{1-\varsigma ; \psi}[a, T] .
$$

From the definition of $C_{1-\varsigma ; \psi}^{n}(a, T]$ with the aid of equations (17), (18), we get

$$
I_{a^{+}}^{1-\eta(1-\theta) ; \psi} \sigma \in C_{1-\varsigma ; \psi}^{1}[a, T] .
$$


Thus $\sigma$ and $I_{a^{+}}^{1-\eta(1-\theta) ; \psi} \sigma$ satisfy assumptions of Lemma 5. Now, we can apply the fractional integral $I_{a^{+}}^{\eta(1-\theta) ; \psi}$ on both sides of (17) and utilize Lemmas 2, 5 to get

$$
\begin{aligned}
I_{a^{+}}^{\eta(1-\theta) ; \psi} D_{a^{+}}^{\zeta ; \psi} y(t) & =\sigma(t)-\frac{I_{a^{+}}^{1-\eta(1-\theta) ; \psi} \sigma(a)}{\Gamma(\eta(1-\theta))} \mathcal{K}_{\psi}^{\eta(1-\theta)}(t, a) \\
& =\sigma(t) .
\end{aligned}
$$

From (5) with $n=1$, equation (19) reduces to

$$
D_{a^{+}}^{\theta, \eta ; \psi} y(t)=\sigma(t), \quad t \in(a, T]
$$

This proves that $y$ also satisfies FDE (7). Undoubtedly, if $y \in C_{1-\varsigma ; \psi}^{\varsigma}[a, T]$ satisfies integral equation (9), then it also fulfills terminal condition (8).

Before we present our main results, we consider that the following assumptions are satisfied:

$\left(H_{1}\right) f_{1}, f_{2}:(a, T] \times \mathbb{R} \rightarrow \mathbb{R}$ are such that

$$
\begin{array}{ll}
f_{1}(\cdot, y(\cdot)) \in C_{1-\varsigma_{1} ; \psi}^{\eta_{1}\left(1-\theta_{1}\right)}[a, T], & y \in C_{1-\varsigma_{1} ; \psi}[a, T], \\
f_{2}(\cdot, x(\cdot)) \in C_{1-\zeta_{2} ; \psi}^{\eta_{2}\left(1-\theta_{2}\right)}[a, T], & y \in C_{1-\varsigma_{2} ; \psi}[a, T] .
\end{array}
$$

$\left(H_{2}\right)$ There exist $L_{1}(>0)$ and $L_{2}(>0)$ such that, for $p, p^{*}, q, q^{*} \in \mathbb{R}, t \in(a, T]$, we have

$$
\begin{aligned}
& \left|f_{1}(t, p)-f_{1}\left(t, p^{*}\right)\right| \leq L_{1}\left|p-p^{*}\right|, \\
& \left|f_{2}(t, q)-f_{2}\left(t, q^{*}\right)\right| \leq L_{2}\left|q-q^{*}\right| .
\end{aligned}
$$

In the forthcoming theorem, by using Theorem 2, we prove the unique solution of a coupled system for $\psi$-Hilfer terminal FDEs (3)-(4). In view of Theorem 3, we get the following lemma.

Lemma 7 Let $i=1,2, \varsigma_{i}=\theta_{i}+\eta_{i}\left(1-\theta_{i}\right)$, where $0<\theta_{i}<1$ and $0 \leq \eta_{i} \leq 1$. If $f_{1}, f_{2}:(a, T] \times$ $\mathbb{R} \rightarrow \mathbb{R}$ two functions such that $f_{1}(\cdot, y) \in C_{1-\varsigma_{1}, \psi}[a, T]$ and $f_{2}(\cdot, x) \in C_{1-\varsigma_{2}, \psi}[a, T]$, then $y \in$ $C_{1-\varsigma_{1}, \psi}^{\varsigma_{1}}(a, T]$ and $x \in C_{1-\varsigma_{2}, \psi}^{\varsigma_{2}}(a, T]$ satisfy the following coupled system offractional integral equations:

$$
\left\{\begin{aligned}
y(t)= & \frac{\mathcal{K}_{\psi}^{S_{1}}(t, a)}{\mathcal{K}_{\psi}^{S_{1}}(T, a)}\left[w_{1}-\frac{1}{\Gamma\left(\theta_{1}\right)} \int_{a}^{T} \mathcal{H}_{\psi}^{\theta_{1}}(T, s) f_{1}(s, x(s)) d s\right] \\
& +\frac{1}{\Gamma\left(\theta_{1}\right)} \int_{a}^{t} \mathcal{H}_{\psi}^{\theta_{1}}(t, s) f_{1}(s, x(s)) d s, \\
x(t)= & \frac{\mathcal{K}_{\psi}^{S_{2}}(t, a)}{\mathcal{K}_{\psi}^{S_{2}}(T, a)}\left[w_{2}-\frac{1}{\Gamma\left(\theta_{2}\right)} \int_{a}^{T} \mathcal{H}_{\psi}^{\theta_{2}}(T, s) f_{2}(s, y(s)) d s\right] \\
& +\frac{1}{\Gamma\left(\theta_{2}\right)} \int_{a}^{t} \mathcal{H}_{\psi}^{\theta_{2}}(t, s) f_{2}(s, y(s)) d s .
\end{aligned}\right.
$$

According to Lemma (7), we consider the operators $\mathcal{N}_{1}: E \rightarrow E$ and $\mathcal{N}_{2}: E \rightarrow E$ defined by

$$
\left\{\begin{array}{l}
\mathcal{N}_{1} x(t)=y(t), \\
\mathcal{N}_{2} y(t)=x(t) .
\end{array}\right.
$$


That is,

$$
\left\{\begin{aligned}
\mathcal{N}_{1} x(t)= & \frac{\mathcal{K}_{\psi}^{\zeta 1}(t, a)}{\mathcal{K}_{\psi}^{S 1}(T, a)}\left[w_{1}-\frac{1}{\Gamma\left(\theta_{1}\right)} \int_{a}^{T} \mathcal{H}_{\psi}^{\theta_{1}}(T, s) f_{1}(s, x(s)) d s\right] \\
& +\frac{1}{\Gamma\left(\theta_{1}\right)} \int_{a}^{t} \mathcal{H}_{\psi}^{\theta_{1}}(t, s) f_{1}(s, x(s)) d s \\
\mathcal{N}_{2} y(t)= & \frac{\mathcal{K}_{\psi}^{S 2}(t, a)}{\mathcal{K}_{\psi}^{S 2}(T, a)}\left[w_{2}-\frac{1}{\Gamma\left(\theta_{2}\right)} \int_{a}^{T} \mathcal{H}_{\psi}^{\theta_{2}}(T, s) f_{2}(s, y(s)) d s\right] \\
& +\frac{1}{\Gamma\left(\theta_{2}\right)} \int_{a}^{t} \mathcal{H}_{\psi}^{\theta_{2}}(t, s) f_{2}(s, y(s)) d s .
\end{aligned}\right.
$$

Therefore, we define $\mathcal{N}: E \times E \rightarrow E \times E$ by

$$
\mathcal{N}(y, x)(t)=\left(\mathcal{N}_{1}(x)(t), \mathcal{N}_{2}(y)(t)\right) .
$$

For the sake of brevity, we set

$$
\begin{aligned}
& \Lambda_{f_{1}}=\left[\frac{2 L_{1} \mathcal{B}\left(\varsigma_{1}, \theta_{1}\right)}{\Gamma\left(\theta_{1}\right)}\right] \mathcal{K}_{\psi}^{\theta_{1}+1}(T, a), \\
& \Delta_{f_{1}}=\frac{w_{1}}{\mathcal{K}_{\psi}^{\zeta 1}(T, a)}+\frac{2 A_{1}}{\Gamma\left(\theta_{1}+1\right)} \mathcal{K}_{\psi}^{2}(T, a), \\
& \Lambda_{f_{2}}=\left[\frac{2 L_{2} \mathcal{B}\left(\varsigma_{2}, \theta_{2}\right)}{\Gamma\left(\theta_{2}\right)}\right] \mathcal{K}_{\psi}^{\theta_{2}+1}(T, a), \\
& \Delta_{f_{2}}=\frac{w_{2}}{\mathcal{K}_{\psi}^{\zeta 2}(T, a)}+\frac{2 A_{2}}{\Gamma\left(\theta_{2}+1\right)} \mathcal{K}_{\psi}^{2}(T, a),
\end{aligned}
$$

where $A_{i}=\max _{t \in[a, T]}\left|f_{i}(t, 0)\right|, i=1,2$.

Now, via Theorems 2, 1, we obtain the existence and uniqueness results of a coupled system for $\psi$-Hilfer FDEs (3)-(4).

Theorem 4 Assume that $\left(H_{1}\right)$ and $\left(H_{2}\right)$ hold. If $\Lambda_{f_{1}}<1$ and $\Lambda_{f_{2}}<1$, then $\psi$-Hilfer coupled system (3)-(4) has a unique solution in $E^{\varsigma} \times E^{\varsigma} \subset E^{\theta, \eta} \times E^{\theta, \eta}$, where $E^{\varsigma}:=C_{1-\varsigma ; \psi}^{\varsigma}[a, T]$ and $E^{\theta, \eta}:=C_{1-\varsigma ; \psi}^{\theta, \eta}[a, T]$.

Proof Define the closed, bounded, convex, and nonempty set

$$
\mathcal{S}_{R}=\left\{(y, x) \in E \times E:\|(y, x)\|_{E \times E} \leq R\right\} \subset E \times E,
$$

with

$$
R \geq \max \left\{\frac{\Delta_{f_{1}}}{1-\Lambda_{f_{1}}}, \frac{\Delta_{f_{2}}}{1-\Lambda_{f_{2}}}\right\} .
$$

The analysis of proof will be presented in three steps.

$\operatorname{Step}(1): \mathcal{N S}_{R} \subset \mathcal{S}_{R}$.

Let $(y, x) \in \mathcal{S}_{R}$ and $t \in(a, T]$. Then

$$
\begin{aligned}
& \left|[\psi(t)-\psi(a)]^{1-\varsigma_{1}}\left(\mathcal{N}_{1} x\right)(t)\right| \\
& \quad \leq \frac{1}{\mathcal{K}_{\psi}^{\varsigma_{1}}(T, a)}\left[w_{1}+\frac{1}{\Gamma\left(\theta_{1}\right)} \int_{a}^{T} \mathcal{H}_{\psi}^{\theta_{1}}(T, s)\left|f_{1}(s, x(s))\right| d s\right]
\end{aligned}
$$




$$
\begin{aligned}
& \quad+\frac{1}{\mathcal{K}_{\psi}^{S_{1}}(t, a)} \frac{1}{\Gamma\left(\theta_{1}\right)} \int_{a}^{t} \mathcal{H}_{\psi}^{\theta_{1}}(t, s)\left|f_{1}(s, x(s))\right| d s \\
& \leq \frac{1}{\mathcal{K}_{\psi}^{S_{1}}(T, a)}\left[w_{1}+\frac{1}{\Gamma\left(\theta_{1}\right)} \int_{a}^{T} \mathcal{H}_{\psi}^{\theta_{1}}(T, s)\right. \\
& \left.\quad \times\left[\left|f_{1}(s, x(s))-f_{1}(s, 0)\right|+\left|f_{1}(s, 0)\right|\right] d s\right] \\
& \quad+\frac{1}{\mathcal{K}_{\psi}^{S_{1}}(t, a)} \frac{1}{\Gamma\left(\theta_{1}\right)} \int_{a}^{t} \mathcal{H}_{\psi}^{\theta_{1}}(t, s) \\
& \quad \times\left[\left|f_{1}(s, x(s))-f_{1}(s, 0)\right|+\left|f_{1}(s, 0)\right|\right] d s .
\end{aligned}
$$

Thanks to hypothesis $\left(H_{2}\right)$, for $(y, x) \in \mathcal{S}_{R}, t \in(a, T]$, we have

$$
\begin{aligned}
\left|[\psi(t)-\psi(a)]^{1-\varsigma_{1}}\left(\mathcal{N}_{1} x\right)(t)\right| & \\
\leq & \frac{1}{\mathcal{K}_{\psi}^{S_{1}}(T, a)}\left[w_{1}+\frac{1}{\Gamma\left(\theta_{1}\right)} \int_{a}^{T} \mathcal{H}_{\psi}^{\theta_{1}}(T, s)\left(L_{1}(\psi(s)-\psi(a))^{1-\varsigma_{1}}\|x\|_{E}+A_{1}\right) d s\right] \\
& +\frac{1}{\mathcal{K}_{\psi}^{S_{1}}(t, a)} \frac{1}{\Gamma\left(\theta_{1}\right)} \int_{a}^{t} \mathcal{H}_{\psi}^{\theta_{1}}(t, s)\left(L_{1}(\psi(s)-\psi(a))^{\varsigma_{1}-1}\|x\|_{E}+A_{1}\right) d s \\
\leq & \frac{1}{\mathcal{K}_{\psi}^{S_{1}}(T, a)}\left[w_{1}+L_{1} R \frac{\Gamma\left(\varsigma_{1}\right)}{\Gamma\left(\varsigma_{1}+\theta_{1}\right)} \mathcal{K}_{\psi}^{\theta_{1}+\varsigma_{1}}(T, a)+\frac{A_{1}}{\Gamma\left(\theta_{1}+1\right)} \mathcal{K}_{\psi}^{\theta_{1}+1}(T, a)\right] \\
& +\frac{1}{\mathcal{K}_{\psi}^{S_{1}}(t, a)}\left[L_{1} R \frac{\Gamma\left(\varsigma_{1}\right)}{\Gamma\left(\varsigma_{1}+\theta_{1}\right)} \mathcal{K}_{\psi}^{\theta_{1}+1}(t, a)+\frac{A_{1}}{\Gamma\left(\theta_{1}+1\right)} \mathcal{K}_{\psi}^{\theta_{1}+1}(t, a)\right] \\
\leq & \frac{w_{1}}{\mathcal{K}_{\psi}^{S_{1}}(T, a)}+\frac{2 A_{1}}{\Gamma\left(\theta_{1}+1\right)} \mathcal{K}_{\psi}^{2}(T, a)+2 L_{1} R \frac{\mathcal{B}\left(\varsigma_{1}, \theta_{1}\right)}{\Gamma\left(\theta_{1}\right)} \mathcal{K}_{\psi}^{\theta_{1}+1}(T, a),
\end{aligned}
$$

where we used the fact that

$$
\frac{1}{\mathcal{K}_{\psi}^{S_{1}}(t, a)} \leq \frac{1}{\mathcal{K}_{\psi}^{\theta_{1}}(t, a)} \quad \text { for } \varsigma_{1} \geq \theta_{1}
$$

and

$$
\mathcal{K}_{\psi}^{\varsigma_{1}}(t, a) \leq 1 \quad \text { for } 0<\varsigma_{1} \leq 1
$$

Hence, inequality (23) becomes

$$
\left|[\psi(t)-\psi(a)]^{1-\varsigma_{1}}\left(\mathcal{N}_{1} x\right)(t)\right| \leq \Lambda_{f_{1}}+\Delta_{f_{1}} R,
$$

which leads to

$$
\left\|\mathcal{N}_{1} x\right\|_{E} \leq \Lambda_{f_{1}}+\Delta_{f_{1}} R \leq R
$$

Similarly, we can get that

$$
\left\|\mathcal{N}_{2} y\right\|_{E} \leq \Lambda_{f_{2}}+\Delta_{f_{2}} R \leq R
$$


It follows from (24) and (25) that

$$
\|\mathcal{N}(y, x)\|_{E \times E}=\max \left(\left\|\mathcal{N}_{1} x\right\|_{E},\left\|\mathcal{N}_{2} y\right\|_{E}\right) \leq \max (R, R)=R .
$$

This proves $\mathcal{N} \mathcal{S}_{R} \subset \mathcal{S}_{R}$.

Step(2): The operator $\mathcal{N}$ is a contraction.

Let $(y, x),\left(y^{*}, x^{*}\right) \in \mathcal{S}_{R}$ and $t \in(a, T]$. Applying $\left(H_{2}\right)$ we have

$$
\begin{aligned}
\left|[\psi(t)-\psi(a)]^{1-\varsigma_{1}}\left[\left(\mathcal{N}_{1} x\right)(t)-\left(\mathcal{N}_{1} x^{*}\right)(t)\right]\right| \\
\leq \frac{1}{\mathcal{K}_{\psi}^{\zeta 1}(T, a)} \frac{1}{\Gamma\left(\theta_{1}\right)} \int_{a}^{T} \mathcal{H}_{\psi}^{\theta_{1}}(T, s)\left|f_{1}(s, x(s))-f_{1}\left(s, x^{*}(s)\right)\right| d s \\
\quad+\frac{1}{\mathcal{K}_{\psi}^{\zeta 1}(t, a)} \frac{1}{\Gamma\left(\theta_{1}\right)} \int_{a}^{t} \mathcal{H}_{\psi}^{\theta_{1}}(t, s)\left|f_{1}(s, x(s))-f_{1}\left(s, x^{*}(s)\right)\right| d s \\
\leq \frac{1}{\mathcal{K}_{\psi}^{\zeta 1}(T, a)} L_{1}\left\|x-x^{*}\right\|_{E}\left(I_{a^{+}}^{\theta_{1}, \psi} \mathcal{K}_{\psi}^{\zeta 1}(s, a)\right)(T) \\
\quad+\frac{1}{\mathcal{K}_{\psi}^{\zeta 1}(t, a)} L_{1}\left\|x-x^{*}\right\|_{E}\left(I_{a^{+}}^{\theta_{1}, \psi} \mathcal{K}_{\psi}^{\zeta_{1}}(s, a)\right)(t) \\
\leq 2 L_{1} \frac{\mathcal{B}\left(\varsigma_{1}, \theta_{1}\right)}{\Gamma\left(\theta_{1}\right)} \mathcal{K}_{\psi}^{\theta_{1}+1}(T, a)\left\|x-x^{*}\right\|_{E},
\end{aligned}
$$

which implies

$$
\left\|\left(\mathcal{N}_{1} x\right)-\left(\mathcal{N}_{1} x^{*}\right)\right\|_{E} \leq \Lambda_{f_{1}}\left\|x-x^{*}\right\|_{E} .
$$

By the same technique, we can also get

$$
\left\|\left(\mathcal{N}_{2} y\right)-\left(\mathcal{N}_{2} y^{*}\right)\right\|_{E} \leq \Lambda_{f_{2}}\left\|x-x^{*}\right\|_{E} .
$$

In view of the conditions $\Lambda_{f_{1}}<1$ and $\Lambda_{f_{2}}<1$, we get

$$
\left\|\mathcal{N}(y, x)-\mathcal{N}\left(y^{*}, x^{*}\right)\right\|_{E \times E}<\left\|(y, x)-\left(y^{*}, x^{*}\right)\right\|_{E \times E}
$$

Thus $\mathcal{N}$ is a contraction mapping. In accordance with Theorem $2, \mathcal{N}$ has a unique fixed point $(\widehat{y}, \widehat{x}) \in E \times E$.

Step (3): We show that such a fixed point $(\widehat{y}, \widehat{x}) \in C_{1-\varsigma_{1} ; \psi}[a, T] \times C_{1-\varsigma_{2} ; \psi}[a, T]$ is actually in $C_{1-\varsigma_{1} ; \psi}^{\varsigma_{1}}[a, T] \times C_{1-\varsigma_{2} ; \psi}^{\varsigma_{2}}[a, T]$. Since $\widehat{y}$ and $\widehat{x}$ are the unique fixed points of $\mathcal{N}_{1}$ and $\mathcal{N}_{2}$ in $C_{1-\varsigma_{1} ; \psi}[a, T] \times C_{1-\varsigma_{2} ; \psi}[a, T]$ respectively, then for $t \in(a, T]$, we have

$$
\left\{\begin{aligned}
\widehat{y}(t)= & \frac{\mathcal{K}_{\psi}^{S 1}(t, a)}{\mathcal{K}_{\psi}^{S 1}(T, a)}\left[w_{1}-\frac{1}{\Gamma\left(\theta_{1}\right)} \int_{a}^{T} \mathcal{H}_{\psi}^{\theta_{1}}(T, s) f_{1}(s, \widehat{x}(s)) d s\right] \\
& +\frac{1}{\Gamma\left(\theta_{1}\right)} \int_{a}^{t} \mathcal{H}_{\psi}^{\theta_{1}}(t, s) f_{1}(s, \widehat{x}(s)) d s, \\
\widehat{x}(t)= & \frac{\mathcal{K}_{\psi}^{S 2}(t, a)}{\mathcal{K}_{\psi}^{S 2}(T, a)}\left[w_{2}-\frac{1}{\Gamma\left(\theta_{2}\right)} \int_{a}^{T} \mathcal{H}_{\psi}^{\theta_{2}}(T, s) f_{2}(s, \widehat{y}(s)) d s\right] \\
& +\frac{1}{\Gamma\left(\theta_{2}\right)} \int_{a}^{t} \mathcal{H}_{\psi}^{\theta_{2}}(t, s) f_{2}(s, \widehat{y}(s)) d s .
\end{aligned}\right.
$$


Multiplying both sides of the last system by $D_{a^{+}}^{\varsigma_{1}, \psi}, D_{a^{+}}^{\varsigma_{2}, \psi}$ respectively, it follows from Lemmas 6 and 3 that

$$
\left\{\begin{array}{l}
D_{a^{+}}^{\zeta_{1}, \psi} \widehat{y}(t)=D_{a^{+}}^{\zeta_{1}, \psi} I_{a^{+}}^{\theta_{1} ; \psi} f_{1}(s, \widehat{x}(s))(t)=D_{a^{+}}^{\eta_{1}\left(1-\theta_{1}\right) ; \psi} f_{1}(s, \widehat{x}(s)), \\
D_{a^{+}}^{\zeta_{2}, \psi} \widehat{x}(t)=D_{a^{+}}^{\zeta_{2}, \psi} I_{a^{+}}^{\theta_{2} ; \psi} f_{2}(s, \widehat{y}(s))(t)=D_{a^{+}}^{\eta_{2}\left(1-\theta_{2}\right) ; \psi} f_{2}(s, \widehat{y}(s)) .
\end{array}\right.
$$

Since $\varsigma_{i} \geq \theta_{i}(i=1,2)$ and by $\left(H_{1}\right)$, we get

$$
\left\{\begin{array}{l}
D_{a^{+}}^{\eta_{1}\left(1-\theta_{1}\right) ; \psi} f_{1}(s, \widehat{x}(s)) \in C_{1-\varsigma_{1} ;}[a, T] \\
D_{a^{+}}^{\eta_{2}\left(1-\theta_{2}\right) ; \psi} f_{2}(s, \widehat{y}(s)) \in C_{1-\varsigma_{2} ; \psi}[a, T] .
\end{array}\right.
$$

Hence, $D_{a^{+}}^{\varsigma_{1}, \psi} \hat{y} \in C_{1-\varsigma_{1} ; \psi}[a, T]$ and $D_{a^{+}}^{\varsigma_{2}, \psi} \widehat{x} \in C_{1-\varsigma_{2} ; \psi}[a, T]$, it follows from the definition of $C_{1-\varsigma_{i} ; \psi}^{\varsigma_{i}}[a, T](i=1,2)$ that $\hat{y} \in C_{1-\varsigma_{1} ; \psi}^{\varsigma_{1}}[a, T]$ and $\widehat{x} \in C_{1-\varsigma_{2} ; \psi}^{\varsigma_{2}}[a, T]$. As a sequel to the steps outlined above, we infer that the $\psi$-Hilfer coupled system (3)-(4) has a unique solution in $C_{1-\varsigma_{1} ; \psi}^{\varsigma_{1}}[a, T] \times C_{1-\varsigma_{2} ; \psi}^{\varsigma_{2}}[a, T]$.

We exhibit now the next result, which relies on Theorem 1.

Theorem 5 Assume that $\left(H_{1}\right)-\left(H_{2}\right)$ hold. Then the $\psi$-Hilfer coupled system (3)-(4) has at least one solution.

Proof Let $\mathcal{K} \subset \mathcal{S}_{R} \subseteq E \times E$ be a bounded set, and we define the operators $\mathcal{F}_{1}, \mathcal{G}_{1}: E \rightarrow E$ and $\mathcal{F}_{2}, \mathcal{G}_{2}: E \rightarrow E$ by

$$
\begin{aligned}
& \left(\mathcal{F}_{1} x\right)(t)=\frac{1}{\Gamma\left(\theta_{1}\right)} \int_{a}^{t} \mathcal{H}_{\psi}^{\theta_{1}}(t, s) f_{1}(s, x(s)) d s \\
& \left(\mathcal{G}_{1} x\right)(t)=\frac{\mathcal{K}_{\psi}^{S_{1}}(t, a)}{\mathcal{K}_{\psi}^{S_{1}}(T, a)}\left[w_{1}-\frac{1}{\Gamma\left(\theta_{1}\right)} \int_{a}^{T} \mathcal{H}_{\psi}^{\theta_{1}}(T, s) f_{1}(s, x(s)) d s\right] \\
& \left(\mathcal{F}_{2} y\right)(t)=\frac{1}{\Gamma\left(\theta_{2}\right)} \int_{a}^{t} \mathcal{H}_{\psi}^{\theta_{2}}(t, s) f_{2}(s, y(s)) d s \\
& \left(\mathcal{G}_{2} y\right)(t)=\frac{\mathcal{K}_{\psi}^{S_{2}}(t, a)}{\mathcal{K}_{\psi}^{S_{2}}(T, a)}\left[w_{2}-\frac{1}{\Gamma\left(\theta_{2}\right)} \int_{a}^{T} \mathcal{H}_{\psi}^{\theta_{2}}(T, s) f_{2}(s, y(s)) d s\right] .
\end{aligned}
$$

From the above-mentioned operators, we are able to write $\mathcal{N}_{1}=\mathcal{F}_{1}+\mathcal{G}_{1}$ and $\mathcal{N}_{2}=\mathcal{F}_{2}+\mathcal{G}_{2}$. Thus, the operator $\mathcal{N}$ can be expressed as

$$
\mathcal{N}=\mathcal{F}+\mathcal{G} \text { such that } \mathcal{F}(y, x)=\left(\mathcal{F}_{1} x, \mathcal{F}_{2} y\right) \text { and } \mathcal{G}(y, x)=\left(\mathcal{G}_{1} x, \mathcal{G}_{2} y\right) \text {. }
$$

The proof will be divided into several stages as follows:

Stage(1): $\mathcal{N}$ is continuous.

The continuity of $f_{1}$ and $f_{2}$ implies the continuity of $\mathcal{N}$.

Stage (2): $\mathcal{F}(\mathcal{K})$ is uniformly bounded.

Let $(y, x) \in \mathcal{K}, t \in(a, T]$. Then, by using $\left(H_{2}\right)$, we have

$$
\left\|\mathcal{F}_{1} x\right\|_{E}=\max _{t \in[a, T]}\left|[\psi(t)-\psi(a)]^{1-\varsigma_{1}}\left(\mathcal{F}_{1} x\right)(t)\right|
$$




$$
\begin{aligned}
& \leq \max _{t \in[a, T]} \frac{1}{\mathcal{K}_{\psi}^{S_{1}}(t, a)} \frac{1}{\Gamma\left(\theta_{1}\right)} \int_{a}^{t} \mathcal{H}_{\psi}^{\theta_{1}}(t, s) f_{1}(s, x(s)) d s \\
& \leq \max _{t \in[a, T]} \frac{1}{\mathcal{K}_{\psi}^{S_{1}}(t, a)} \frac{1}{\Gamma\left(\theta_{1}\right)} \int_{a}^{t} \mathcal{H}_{\psi}^{\theta_{1}}(t, s)\left(\left|f_{1}(s, x(s))-f_{1}(s, 0)\right|+\left|f_{1}(s, 0)\right|\right) d s \\
& \leq \max _{t \in[a, T]} \frac{1}{\mathcal{K}_{\psi}^{S_{1}}(t, a)} \frac{1}{\Gamma\left(\theta_{1}\right)} \int_{a}^{t} \mathcal{H}_{\psi}^{\theta_{1}}(t, s)\left(A_{1}+L_{1}[\psi(s)-\psi(a)]^{S_{1}-1}\|x\|_{E}\right) d s \\
& \leq \max _{t \in[a, T]}\left[\frac{A_{1}}{\Gamma\left(\theta_{1}+1\right)} \mathcal{K}_{\psi}^{2}(t, a)+L_{1} \frac{\mathcal{B}\left(\varsigma_{1}, \theta_{1}\right)}{\Gamma\left(\theta_{1}\right)} \mathcal{K}_{\psi}^{\theta_{1}+1}(t, a)\|x\|_{E}\right] \\
& \leq\left[\frac{A_{1}}{\Gamma\left(\theta_{1}+1\right)}+L_{1} \frac{\mathcal{B}\left(\varsigma_{1}, \theta_{1}\right)}{\Gamma\left(\theta_{1}\right)}\right] \mathcal{K}_{\psi}^{2}(T, a)\|x\|_{E}:=R_{1},
\end{aligned}
$$

where $\mathcal{K}_{\psi}^{\theta_{1}+1}(t, a)<\mathcal{K}_{\psi}^{2}(t, a)$ for $0<\theta_{1}<1$. In the same fashion, we get

$$
\left\|\mathcal{F}_{2} y\right\|_{E} \leq\left[\frac{A_{2}}{\Gamma\left(\theta_{2}+1\right)}+L_{2} \frac{\mathcal{B}\left(\varsigma_{2}, \theta_{2}\right)}{\Gamma\left(\theta_{2}\right)}\right] \mathcal{K}_{\psi}^{2}(T, a)\|y\|_{E}:=R_{2}
$$

which implies

$$
\|\mathcal{F}(y, x)\|_{E \times E} \leq \max \left(R_{1}, R_{2}\right) .
$$

This proves $\mathcal{F}(\mathcal{K})$ is uniformly bounded.

Stage(3): $\mathcal{F}(\mathcal{K})$ is equicontinuous in $\mathcal{K}$.

Let $(y, x) \in \mathcal{K}$ and $t_{1}, t_{2} \in(a, T]$ with $t_{1}<t_{2}$. Then we have

$$
\begin{aligned}
&\left|\left[\psi\left(t_{2}\right)-\psi(a)\right]^{1-\varsigma_{1}}\left(\mathcal{F}_{1} x\right)\left(t_{2}\right)-\left[\psi\left(t_{1}\right)-\psi(a)\right]^{1-\varsigma_{1}}\left(\mathcal{F}_{1} x\right)\left(t_{1}\right)\right| \\
& \leq \frac{1}{\mathcal{K}_{\psi}^{S 1}\left(t_{2}, a\right)} \frac{1}{\Gamma\left(\theta_{1}\right)} \int_{t_{1}}^{t_{2}} \mathcal{H}_{\psi}^{\theta_{1}}\left(t_{2}, s\right)\left|f_{1}(s, x(s))\right| d s \\
&+\frac{1}{\Gamma\left(\theta_{1}\right)}\left|\int_{a}^{t_{1}} \frac{1}{\mathcal{K}_{\psi}^{S_{1}}\left(t_{2}, a\right)} \mathcal{H}_{\psi}^{\theta_{1}}\left(t_{2}, s\right)-\frac{1}{\mathcal{K}_{\psi}^{S_{1}}\left(t_{1}, a\right)} \mathcal{H}_{\psi}^{\theta_{1}}\left(t_{1}, s\right)\right|\left|f_{1}(s, x(s))\right| d s \\
& \leq \frac{1}{\mathcal{K}_{\psi}^{S_{1}}\left(t_{2}, a\right)}\left(I_{t_{1}}^{\theta_{1}, \psi}[\psi(s)-\psi(a)]^{\varsigma_{1}-1}\right)\left(t_{2}\right)|| f_{1}(\cdot, x(\cdot)) \|_{E} \\
&+\mid \frac{1}{\mathcal{K}_{\psi}^{S_{1}}\left(t_{2}, a\right)}\left(I_{a}^{\theta_{1}, \psi}[\psi(s)-\psi(a)]^{\varsigma_{1}-1}\right)\left(t_{2}\right) \\
&-\frac{1}{\mathcal{K}_{\psi}^{S_{1}}\left(t_{1}, a\right)}\left(I_{a}^{\theta_{1}, \psi}[\psi(s)-\psi(a)]^{\varsigma_{1}-1}\right)\left(t_{1}\right) \mid\left\|f_{1}(\cdot, x(\cdot))\right\|_{E} \\
& \leq \frac{1}{\mathcal{K}_{\psi}^{S_{1}}\left(t_{2}, a\right)} \frac{\mathcal{B}\left(\varsigma_{1}, \theta_{1}\right)}{\Gamma\left(\theta_{1}\right)}\left(\left[\psi\left(t_{2}\right)-\psi\left(t_{1}\right)\right]^{\theta_{1}+\varsigma_{1}-1}\right)\left\|f_{1}(\cdot, x(\cdot))\right\|_{E} \\
&+\mid \frac{1}{\mathcal{K}_{\psi}^{S 1}\left(t_{2}, a\right)} \frac{\mathcal{B}\left(\varsigma_{1}, \theta_{1}\right)}{\Gamma\left(\theta_{1}\right)}\left(\left[\psi\left(t_{2}\right)-\psi(a)\right]^{\theta_{1}+\varsigma_{1}-1}\right) \\
&-\frac{1}{\mathcal{K}_{\psi}^{S_{1}}\left(t_{1}, a\right)} \frac{\mathcal{B}\left(\varsigma_{1}, \theta_{1}\right)}{\Gamma\left(\theta_{1}\right)}\left(\left[\psi\left(t_{1}\right)-\psi(a)\right]^{\theta_{1}+\varsigma_{1}-1}\right) \mid\left\|f_{1}(\cdot, x(\cdot))\right\|_{E}
\end{aligned}
$$




$$
\begin{aligned}
\leq & \frac{\mathcal{B}\left(\varsigma_{1}, \theta_{1}\right)}{\Gamma\left(\theta_{1}\right)}\left\|f_{1}(\cdot, x(\cdot))\right\|_{E}\left[\frac{\left[\psi\left(t_{2}\right)-\psi\left(t_{1}\right)\right]^{\theta_{1}+\varsigma_{1}-1}}{\mathcal{K}_{\psi}^{S_{1}}\left(t_{2}, a\right)}\right. \\
& \left.+\left|\left[\psi\left(t_{2}\right)-\psi(a)\right]^{\theta_{1}}-\left[\psi\left(t_{1}\right)-\psi(a)\right]^{\theta_{1}}\right|\right],
\end{aligned}
$$

which leads to

$$
\left\|\left(\mathcal{F}_{1} x\right)\left(t_{2}\right)-\left(\mathcal{F}_{1} x\right)\left(t_{1}\right)\right\|_{E} \rightarrow 0, \quad \text { as } t_{2} \rightarrow t_{1} .
$$

Again applying the same reasoning, we have

$$
\left\|\mathcal{F}_{2} y\left(t_{2}\right)-\mathcal{F}_{2} y\left(t_{1}\right)\right\|_{E} \rightarrow 0, \quad \text { as } t_{2} \rightarrow t_{1} .
$$

This exhibits that $\mathcal{F}(\mathcal{K})$ is equicontinuous. Stages $1-3$ show that $\mathcal{F}$ is relatively compact on $\mathcal{K}$. By $E\left(=C_{1-\varsigma ; \psi}\right)$ type Arzelá-Ascoli theorem, $\mathcal{F}$ is compact on $\mathcal{K}$.

Stage(4): $\mathcal{Q}$ is a contraction operator.

Let $(y, x),\left(y^{*}, x^{*}\right) \in E \times E$ and $t \in(a, T]$. Then, by applying $\left(H_{2}\right)$, we easily get

$$
\left\|\left(\mathcal{G}_{1} x\right)-\left(\mathcal{G}_{1} x^{*}\right)\right\|_{E} \leq \Lambda_{f_{1}}\left\|x-x^{*}\right\|_{E}
$$

and

$$
\left\|\left(\mathcal{G}_{2} y\right)-\left(\mathcal{G}_{2} y^{*}\right)\right\|_{E} \leq \Lambda_{f_{2}}\left\|y-y^{*}\right\|_{E} .
$$

Since $\Lambda_{f_{1}}, \Lambda_{f_{2}}<1, \mathcal{G}$ is a contraction mapping. Using Theorem 1 , we see that $\mathcal{N}$ has at least one fixed point, which is the corresponding solution of $\psi$-Hilfer coupled system (3)-(4).

\section{Ulam-Hyers stability}

This part is devoted to proving the Ulam-Hyers (U-H) and generalized Ulam-Hyers (GU-H) stability of solution to the $\psi$-Hilfer coupled system (3)-(4).

Definition 5 The $\psi$-Hilfer coupled system (3)-(4) is U-H stable if there exists $\lambda=$ $\left(\lambda_{f_{1}}, \lambda_{f_{2}}\right)>0$ with the following property: For some $\epsilon=\left(\epsilon_{1}, \epsilon_{2}\right)>0$ and each $\left.\widetilde{y}, \widetilde{x}\right) \in E \times E$, if

$$
\begin{aligned}
& \left|D_{a^{+}}^{\theta_{1}, \eta_{1}, \psi} \widetilde{y}(t)-f_{1}(t, \tilde{x}(t))\right| \leq \epsilon_{1}, \\
& \left|D_{a^{+}}^{\theta_{2}, \eta_{2}, \psi} \widetilde{x}(t)-f_{2}(t, \tilde{y}(t))\right| \leq \epsilon_{2},
\end{aligned}
$$

then there exists $(y, x) \in E \times E$ satisfying coupled system (3) with the following coupled boundary conditions:

$$
\left\{\begin{array}{l}
y(T)=\tilde{y}(T), \\
x(T)=\widetilde{x}(T),
\end{array}\right.
$$

complying with

$$
\|(\tilde{y}, \tilde{x})-(y, x)\|_{E \times E} \leq \lambda \epsilon .
$$


Definition 6 The $\psi$-Hilfer coupled system (3)-(4) is G-U-H stable if there exists $\varphi=$ $\left(\varphi_{f_{1}}, \varphi_{f_{2}}\right) \in C\left(\mathbb{R}^{+}, \mathbb{R}^{+}\right)$with $\varphi(0)=\left(\varphi_{f_{1}}(0), \varphi_{f_{2}}(0)\right)=(0,0)$ such that for some $\epsilon=\left(\epsilon_{1}, \epsilon_{2}\right)>0$ and for each solution $(\tilde{y}, \tilde{x}) \in E \times E$ of inequalities (27), (28) there exists a solution $(y, x) \in$ $E \times E$ of coupled system (3)-(29) complying with

$$
\|(\widetilde{y}, \widetilde{x})-(y, x)\|_{E \times E} \leq \varphi(\epsilon) .
$$

Remark 1 A function $(\widetilde{y}, \widetilde{x}) \in E \times E$ is a solution of inequalities (27)-(28) if and only if there exists a function $\left(h_{1}, h_{2}\right) \in E \times E$ (where $h_{1}$ depends on solution $\tilde{y}$ and $h_{2}$ depends on solution $\widetilde{x}$ ) such that

(i) $\left|h_{1}(t)\right| \leq \epsilon_{1}$ and $\left|h_{2}(t)\right| \leq \epsilon_{2}$ for $t \in(a, T]$,

(ii) For $t \in(a, T]$,

$$
\left\{\begin{array}{l}
D_{a^{+}}^{\theta_{1}, \eta_{1}, \psi} \widetilde{y}(t)=f_{1}(t, \widetilde{x}(t))+h_{1}(t), \\
D_{a^{+}}^{\theta_{2}, \eta_{2}, \psi} \widetilde{x}(t)=f_{2}(t, \tilde{y}(t))+h_{2}(t) .
\end{array}\right.
$$

Lemma 8 Let $(\widetilde{y}, \widetilde{x}) \in E \times E$ be the solution of inequalities (27)-(28). Then $(y, x) \in E \times E$ is the solution of the following fractional integral inequalities:

$$
\left|\tilde{y}(t)-Z_{\widetilde{x}}-\frac{1}{\Gamma\left(\theta_{1}\right)} \int_{a}^{t} \mathcal{H}_{\psi}^{\theta_{1}}(t, s) f_{1}(s, \widetilde{x}(s)) d s\right| \leq \frac{2 \epsilon_{1} \mathcal{K}_{\psi}^{\theta_{1}+1}(T, a)}{\Gamma\left(\theta_{1}+1\right)}
$$

and

$$
\left|\widetilde{x}(t)-Z_{\widetilde{u}}-\frac{1}{\Gamma\left(\theta_{2}\right)} \int_{a}^{t} \mathcal{H}_{\psi}^{\theta_{2}}(t, s) f_{2}(s, \widetilde{y}(s)) d s\right| \leq \frac{2 \epsilon_{2} \mathcal{K}_{\psi}^{\theta_{2}+1}(T, a)}{\Gamma\left(\theta_{2}+1\right)}
$$

where

$$
Z_{\widetilde{x}}:=\frac{\mathcal{K}_{\psi}^{S 1}(t, a)}{\mathcal{K}_{\psi}^{S_{1}}(T, a)}\left[w_{1}-\frac{1}{\Gamma\left(\theta_{1}\right)} \int_{a}^{T} \mathcal{H}_{\psi}^{\theta_{1}}(T, s) f_{1}(s, \widetilde{x}(s)) d s\right]
$$

and

$$
Z_{\tilde{y}}:=\frac{\mathcal{K}_{\psi}^{S_{2}}(t, a)}{\mathcal{K}_{\psi}^{S_{2}}(T, a)}\left[w_{2}-\frac{1}{\Gamma\left(\theta_{2}\right)} \int_{a}^{T} \mathcal{H}_{\psi}^{\theta_{2}}(T, s) f_{2}(s, \widetilde{y}(s)) d s\right] .
$$

Proof Thanks to Remark 1, we have

$$
\left\{\begin{array}{l}
D_{a^{+}}^{\theta_{1}, \eta_{1}, \psi} \widetilde{y}(t)=f_{1}(t, \tilde{x}(t))+h_{1}(t), \\
D_{a^{+}}^{\theta_{2}, \eta_{2}, \psi} \widetilde{x}(t)=f_{2}(t, \widetilde{y}(t))+h_{2}(t), \\
\widetilde{y}(T)=w_{1}, \quad \widetilde{x}(T)=w_{2} .
\end{array}\right.
$$


Thanks to Theorem 15, the solution of (30) is defined by

$$
\tilde{y}(t)=\left\{\begin{array}{l}
\frac{\mathcal{K}_{\psi}^{S 1}(t, a)}{\mathcal{K}_{\psi}^{S 1}(T, a)}\left[w_{1}-\frac{1}{\Gamma\left(\theta_{1}\right)} \int_{a}^{T} \mathcal{H}_{\psi}^{\theta_{1}}(T, s) f_{1}(s, \widetilde{x}(s)) d s\right] \\
\quad+\frac{\mathcal{K}_{\psi}^{S 1}(t, a)}{\mathcal{K}_{\psi}^{S 1}(T, a)}\left[w_{1}-\frac{1}{\Gamma\left(\theta_{1}\right)} \int_{a}^{T} \mathcal{H}_{\psi}^{\theta_{1}}(T, s) h_{1}(s) d s\right] \\
\quad+\frac{1}{\Gamma\left(\theta_{1}\right)} \int_{a}^{t} \mathcal{H}_{\psi}^{\theta_{1}}(t, s) h_{1}(s) d s \\
\quad+\frac{1}{\Gamma\left(\theta_{1}\right)} \int_{a}^{t} \mathcal{H}_{\psi}^{\theta_{1}}(t, s) f_{1}(s, \widetilde{x}(s)) d s
\end{array}\right.
$$

and

$$
\widetilde{x}(t)=\left\{\begin{array}{l}
\frac{\mathcal{K}_{\psi}^{\varsigma_{2}}(t, a)}{\mathcal{K}_{\psi}^{S_{2}}(T, a)}\left[w_{2}-\frac{1}{\Gamma\left(\theta_{2}\right)} \int_{a}^{T} \mathcal{H}_{\psi}^{\theta_{2}}(T, s) f_{2}(s, \tilde{y}(s)) d s\right] \\
\quad+\frac{\mathcal{K}_{\psi}^{\zeta 2}(t, a)}{\mathcal{K}_{\psi}^{S_{2}}(T, a)}\left[w_{2}-\frac{1}{\Gamma\left(\theta_{1}\right)} \int_{a}^{T} \mathcal{H}_{\psi}^{\theta_{2}}(T, s) h_{1}(s) d s\right] \\
\quad+\frac{1}{\Gamma\left(\theta_{2}\right)} \int_{a}^{t} \mathcal{H}_{\psi}^{\theta_{2}}(t, s) h_{1}(s) d s \\
\quad+\frac{1}{\Gamma\left(\theta_{2}\right)} \int_{a}^{t} \mathcal{H}_{\psi}^{\theta_{2}}(t, s) f_{2}(s, \tilde{y}(s)) d s
\end{array}\right.
$$

It follows from (31) and (32) with using Lemma 8 that

$$
\begin{aligned}
& \left|\tilde{y}(t)-Z_{\widetilde{x}}-\frac{1}{\Gamma\left(\theta_{1}\right)} \int_{a}^{t} \mathcal{H}_{\psi}^{\theta_{1}}(t, s) f_{1}(s, \widetilde{x}(s)) d s\right| \\
& \leq \frac{\mathcal{K}_{\psi}^{S_{1}}(t, a)}{\mathcal{K}_{\psi}^{51}(T, a)} \frac{1}{\Gamma\left(\theta_{1}\right)} \int_{a}^{T} \mathcal{H}_{\psi}^{\theta_{1}}(T, s)\left|h_{1}(s)\right| d s+\frac{1}{\Gamma\left(\theta_{1}\right)} \int_{a}^{t} \mathcal{H}_{\psi}^{\theta_{1}}(t, s)\left|h_{1}(s)\right| d s \\
& \leq \epsilon_{1} \frac{\mathcal{K}_{\psi}^{S_{1}}(t, a)}{\mathcal{K}_{\psi}^{S_{1}}(T, a)} \frac{1}{\Gamma\left(\theta_{1}+1\right)} \mathcal{K}_{\psi}^{\theta_{1}+1}(T, a)+\frac{\epsilon_{1}}{\Gamma\left(\theta_{1}+1\right)} \mathcal{K}_{\psi}^{\theta_{1}+1}(t, a) \\
& \leq \frac{2 \epsilon_{1} \mathcal{K}_{\psi}^{\theta_{1}+1}(T, a)}{\Gamma\left(\theta_{1}+1\right)}
\end{aligned}
$$

where $\mathcal{K}_{\psi}^{\mu}(t, a) \leq \mathcal{K}_{\psi}^{\mu}(T, a)$. Along the same lines, we can also get

$$
\left|\widetilde{x}(t)-Z_{\widetilde{u}}-\frac{1}{\Gamma\left(\theta_{2}\right)} \int_{a}^{t} \mathcal{H}_{\psi}^{\theta_{2}}(t, s) f_{2}(s, \tilde{y}(s)) d s\right| \leq \frac{2 \epsilon_{2} \mathcal{K}_{\psi}^{\theta_{2}+1}(T, a)}{\Gamma\left(\theta_{2}+1\right)} .
$$

Theorem 6 Under the assumptions of Theorem 4 , if $1-\mathcal{C}_{\theta_{1}} \mathcal{C}_{\theta_{2}} \neq 0$, then the $\psi$-Hilfer coupled system (3)-(4) will be $U$ - $H$ and G-U-H stable in $E \times E$, where $\mathcal{C}_{\theta_{1}}:=\frac{\Gamma\left(\varsigma_{1}\right) L_{1}}{\Gamma\left(\varsigma_{1}+\theta_{1}\right)} \mathcal{K}_{\psi}^{\theta_{1}+1}(T, a)$ and $\mathcal{C}_{\theta_{2}}:=\frac{\Gamma\left(\varsigma_{2}\right) L_{2}}{\Gamma\left(\varsigma_{2}+\theta_{2}\right)} \mathcal{K}_{\psi}^{\theta_{2}+1}(T, a)$.

Proof Let $\widetilde{y}, \widetilde{x}) \in E \times E$ be the solution of coupled system $(30)$ and $(y, x) \in E \times E$ be a unique solution of the $\psi$-Hilfer coupled system (3)-(4) with the conditions

$$
\left\{\begin{array}{l}
y(T)=\tilde{y}(T)=w_{1} \\
x(T)=\widetilde{x}(T)=w_{2}
\end{array}\right.
$$

That is,

$$
y(t)=Z_{x}+\frac{1}{\Gamma\left(\theta_{1}\right)} \int_{a}^{t} \mathcal{H}_{\psi}^{\theta_{1}}(t, s) f_{1}(s, x(s)) d s
$$


and

$$
x(t)=Z_{y}+\frac{1}{\Gamma\left(\theta_{2}\right)} \int_{a}^{t} \mathcal{H}_{\psi}^{\theta_{2}}(t, s) f_{2}(s, y(s)) d s .
$$

Due to (33), $Z_{\widetilde{x}}=Z_{x}$ and $Z_{\widetilde{y}}=Z_{y}$. Hence relations (34) and (35) become

$$
y(t)=Z_{\tilde{x}}+\frac{1}{\Gamma\left(\theta_{1}\right)} \int_{a}^{t} \mathcal{H}_{\psi}^{\theta_{1}}(t, s) f_{1}(s, x(s)) d s
$$

and

$$
x(t)=Z_{\widetilde{y}}+\frac{1}{\Gamma\left(\theta_{2}\right)} \int_{a}^{t} \mathcal{H}_{\psi}^{\theta_{2}}(t, s) f_{2}(s, y(s)) d s .
$$

Through Lemma 8, we arrive at

$$
\left|\tilde{y}(t)-Z_{\widetilde{x}}-\frac{1}{\Gamma\left(\theta_{1}\right)} \int_{a}^{t} \mathcal{H}_{\psi}^{\theta_{1}}(t, s) f_{1}(s, \tilde{x}(s)) d s\right| \leq \mathcal{A}_{1} \epsilon_{1}
$$

and

$$
\left|\tilde{x}(t)-Z_{\widetilde{u}}-\frac{1}{\Gamma\left(\theta_{2}\right)} \int_{a}^{t} \mathcal{H}_{\psi}^{\theta_{2}}(t, s) f_{2}(s, \tilde{y}(s)) d s\right| \leq \mathcal{A}_{2} \epsilon_{2},
$$

where $\mathcal{A}_{1}=\frac{2 \mathcal{K}_{\psi}^{\theta_{1}+1}(T, a)}{\Gamma\left(\theta_{1}+1\right)}$ and $\mathcal{A}_{2}=\frac{2 \mathcal{K}_{\psi}^{\theta_{2}+1}(T, a)}{\Gamma\left(\theta_{2}+1\right)}$.

Thus, by $\left(H_{2}\right)$ and inequalities (36), (37), we reach

$$
\begin{aligned}
&|\widetilde{y}(t)-y(t)| \\
&=\left|\widetilde{y}(t)-Z_{\widetilde{x}}-\frac{1}{\Gamma\left(\theta_{1}\right)} \int_{a}^{t} \mathcal{H}_{\psi}^{\theta_{1}}(t, s) f_{1}(s, \widetilde{x}(s)) d s\right| \\
& \quad+\frac{1}{\Gamma\left(\theta_{1}\right)} \int_{a}^{t} \mathcal{H}_{\psi}^{\theta_{1}}(t, s)\left|f_{1}(s, \widetilde{x}(s))-f_{1}(s, x(s))\right| d s \\
& \leq \mathcal{A}_{1} \epsilon_{1}+\frac{1}{\Gamma\left(\theta_{1}\right)} \int_{a}^{t} \mathcal{H}_{\psi}^{\theta_{1}}(t, s) L_{1}|\widetilde{x}(s)-x(s)| d s \\
& \leq \mathcal{A}_{1} \epsilon_{1}+\frac{\Gamma\left(\varsigma_{1}\right) L_{1}}{\Gamma\left(\varsigma_{1}+\theta_{1}\right)} \mathcal{K}_{\psi}^{\theta_{1}+\varsigma_{1}}(t, a)\|\widetilde{x}-x\|_{E},
\end{aligned}
$$

which implies

$$
\|\tilde{y}-y\|_{E} \leq \overline{\mathcal{A}_{1}} \epsilon_{1}+\mathcal{C}_{\theta_{1}}\|\tilde{x}-x\|_{E},
$$

where $\overline{\mathcal{A}_{1}}:=\frac{\mathcal{A}_{1}}{\mathcal{K}_{\psi}^{S 1}(T, a)}$.

Similarly, we have

$$
\|\tilde{x}-x\|_{E} \leq \overline{\mathcal{A}_{2}} \epsilon_{2}+\mathcal{C}_{\theta_{2}}\|\tilde{y}-y\|_{E},
$$


where $\overline{\mathcal{A}_{2}}:=\frac{\mathcal{A}_{2}}{\mathcal{K}_{\psi}^{\zeta_{2}}(T, a)}$. Inequalities (38) and (39) can be rewritten again as follows:

$$
\left\{\begin{array}{l}
\|\tilde{y}-y\|_{E}-\mathcal{C}_{\theta_{1}}\|\tilde{x}-x\|_{E} \leq \overline{\mathcal{A}_{1}} \epsilon_{1} \\
\|\tilde{x}-x\|_{E}-\mathcal{C}_{\theta_{2}}\|\tilde{y}-y\|_{E} \leq \overline{\mathcal{A}_{2}} \epsilon_{2}
\end{array}\right.
$$

Now, we will represent the relations in (40) as matrices as follows:

$$
\left(\begin{array}{cc}
1 & -\mathcal{C}_{\theta_{1}} \\
-\mathcal{C}_{\theta_{2}} & 1
\end{array}\right)\left(\begin{array}{c}
\|\tilde{y}-y\|_{E} \\
\|\tilde{x}-x\|_{E}
\end{array}\right) \leq\left(\begin{array}{l}
\overline{\mathcal{A}_{1}} \epsilon_{1} \\
\overline{\mathcal{A}_{2}} \epsilon_{2}
\end{array}\right)
$$

After simple computations of the above inequality, we can write

$$
\left(\begin{array}{c}
\|\tilde{y}-y\|_{E} \\
\|\tilde{x}-x\|_{E}
\end{array}\right) \leq\left(\begin{array}{cc}
\frac{1}{\Delta} & \frac{\mathcal{C}_{\theta_{1}}}{\Delta} \\
\frac{\mathcal{C}_{\theta_{2}}}{\Delta} & \frac{1}{\Delta}
\end{array}\right) \times\left(\begin{array}{l}
\overline{\mathcal{A}_{1}} \epsilon_{1} \\
\overline{\mathcal{A}_{2}} \epsilon_{2}
\end{array}\right)
$$

where $\Delta=1-\mathcal{C}_{\theta_{1}} \mathcal{C}_{\theta_{2}} \neq 0$. This leads to

$$
\begin{aligned}
\|\tilde{y}-y\|_{E} & \leq \frac{\overline{\mathcal{A}_{1}}}{\Delta} \epsilon_{1}+\frac{\mathcal{C}_{\theta_{1}} \overline{\mathcal{A}_{2}}}{\Delta} \epsilon_{2}, \\
\|\widetilde{x}-x\|_{E} & \leq \frac{\mathcal{C}_{\theta_{2}} \overline{\mathcal{A}_{1}}}{\Delta} \epsilon_{1}+\frac{\overline{\mathcal{A}_{2}}}{\Delta} \epsilon_{2} .
\end{aligned}
$$

By compiling the above two inequalities, we get

$$
\|\tilde{y}-y\|_{E}+\|\tilde{x}-x\|_{E} \leq\left(\frac{\overline{\mathcal{A}_{1}}+\mathcal{C}_{\theta_{2}} \overline{\mathcal{A}_{1}}}{\Delta}\right) \epsilon_{1}+\left(\frac{\overline{\mathcal{A}_{2}}+\mathcal{C}_{\theta_{1}} \overline{\mathcal{A}_{2}}}{\Delta}\right) \epsilon_{2}
$$

For $\epsilon=\max \left\{\epsilon_{1}, \epsilon_{2}\right\}$ and

$$
\lambda=\left(\frac{\overline{\mathcal{A}_{1}}+\mathcal{C}_{\theta_{2}} \overline{\mathcal{A}_{1}}+\overline{\mathcal{A}_{2}}+\mathcal{C}_{\theta_{1}} \overline{\mathcal{A}_{2}}}{\Delta}\right)
$$

we obtain

$$
\|(\widetilde{y}, \tilde{x})-(y, x)\|_{E \times E} \leq \lambda \epsilon
$$

This proves that the $\psi$-Hilfer coupled system (3)-(4) is U-H stable.

Moreover, we could put into writing inequality (41) as

$$
\|(\widetilde{y}, \widetilde{x})-(y, x)\|_{E \times E} \leq \varphi(\epsilon)
$$

where $\varphi(\epsilon)=\lambda \epsilon$ with $\varphi(0)=0$. This shows that the $\psi$-Hilfer coupled system (3)-(4) is G-U-H stable.

\section{Examples}

Here, we provide some illustrative examples to validate the obtained results. 
Example 1 Consider the following coupled system of TVP for $\psi$-Hilfer FDE:

$$
\begin{cases}D_{0^{+}}^{\frac{1}{2}, 0 ; e^{\frac{t}{3}}} y(t)=\frac{1}{10 e^{t+1}} \frac{|x(t)|}{1+|x(t)|}+\frac{1}{\sqrt{t+1}}, & t \in(0,1], \\ D_{0^{+}}^{\frac{1}{2}, 0 ; e^{\frac{t}{3}}} x(t)=\frac{1}{(t+6)^{2}} \frac{|y(t)|}{1+|y(t)|}+\frac{1}{2 \sqrt{t+1}}, & t \in(0,1], \\ y(1)=1, \quad x(1)=2 . & \end{cases}
$$

Set

$$
f_{1}(t, u)=\frac{1}{10 e^{t+1}} \frac{u}{1+u}+\frac{1}{\sqrt{t+1}}, \quad u, v \in \mathbb{R}, t \in(0,1]
$$

and

$$
f_{2}(t, v)=\frac{1}{(t+6)^{2}} \frac{v}{1+v}+\frac{1}{2 \sqrt{t+1}} \quad u, v \in \mathbb{R}, t \in(0,1] .
$$

Then

$$
\begin{aligned}
& C_{1-\varsigma_{1} ; \psi}^{\eta_{1}\left(1-\theta_{1}\right)}[0,1]=C_{\frac{1}{2} ; e^{\frac{t}{3}}}^{0}[0,1]=\left\{f_{1}:(0,1] \times \mathbb{R} \rightarrow \mathbb{R} ;\left(e^{\frac{t}{3}}-1\right)^{\frac{1}{2}} f_{1} \in C[0,1]\right\}, \\
& C_{1-\varsigma_{2} ; \psi}^{\eta_{2}\left(1-\theta_{2}\right)}[0,1]=C_{\frac{1}{2} ; e^{\frac{t}{3}}}^{0}[0,1]=\left\{f_{2}:(0,1] \times \mathbb{R} \rightarrow \mathbb{R} ;\left(e^{\frac{t}{3}}-1\right)^{\frac{1}{2}} f_{2} \in C[0,1]\right\},
\end{aligned}
$$

with $\theta_{1}=\theta_{2}=\frac{1}{2}, \eta_{1}=\eta_{2}=0, \varsigma_{1}=\varsigma_{2}=\frac{1}{2}, \psi(t)=e^{\frac{t}{3}}$, and $(a, T]=(0,1]$. Evidently, the functions $f_{1}, f_{2} \in C_{\frac{1}{2} ; e^{\frac{t}{3}}}[0,1]$. So, hypothesis $\left(H_{1}\right)$ holds. For $t \in(0,1]$ and $u, u^{*}, v, v^{*} \in \mathbb{R}$, we have

$$
\begin{aligned}
& \left|f_{1}(t, u)-f_{1}\left(t, u^{*}\right)\right| \leq \frac{1}{10 e}\left|u-u^{*}\right|, \\
& \left|f_{2}(t, v)-f_{2}\left(t, v^{*}\right)\right| \leq \frac{1}{36}\left|v-v^{*}\right| .
\end{aligned}
$$

Hence, hypothesis $\left(H_{2}\right)$ is satisfied with $L_{1}=\frac{1}{10 e}$ and $L_{2}=\frac{1}{36}$. By some straightforward calculations, we find that $A_{1}=1, A_{2}=\frac{1}{2}, \Lambda_{f_{1}} \approx 0.08<1, \Lambda_{f_{2}} \approx 0.06<1, \Delta_{f_{1}} \approx 1.5$, and $\Delta_{f_{2}} \approx 1.7$. Thus all the assumptions in Theorem 4 are satisfied. An application of Theorem 4 shows that problem (42) has a unique solution in $C_{\frac{1}{2}, e^{\frac{t}{3}}}^{\frac{1}{2}}[0,1]$. However, $\Delta=$ $1-\mathcal{C}_{\theta_{1}} \mathcal{C}_{\theta_{1}}=1-\frac{\pi(\sqrt[3]{e}-1)}{360 e} \neq 0$, where $\mathcal{C}_{\theta_{1}}=\frac{\sqrt{\pi(\sqrt[3]{e}-1)}}{10 e}$ and $\mathcal{C}_{\theta_{2}}=\frac{\sqrt{\pi(\sqrt[3]{e}-1)}}{36}$. Therefore, from Theorem 6, coupled system (42) is U-H and G-U-H stable.

Example 2 A particular case, for $\theta_{1}=\theta_{2}=\frac{1}{2}, \eta_{1}=\eta_{2}=1$, and $\psi(t)=t$, the coupled system for $\psi$-Hilfer FDE (42) reduces to the following coupled system for Caputo FDE:

$$
\begin{cases}{ }^{C} D_{0^{+}}^{\frac{1}{2}} y(t)=\frac{1}{10 e^{t+1}} \frac{|x(t)|}{1+|x(t)|}+\frac{1}{\sqrt{t+1}}, & t \in[0,1], \\ { }^{C} D_{0^{+}}^{\frac{1}{2}} x(t)=\frac{1}{(t+2)^{2}} \frac{|y(t)|}{1+|y(t)|}+\frac{1}{2 \sqrt{t+1}}, & t \in[0,1], \\ y(1)=1, \quad x(1)=2 . & \end{cases}
$$

Clearly, the function $f_{1}, f_{2} \in C[0,1]$. Hence $\left(H_{1}\right)$ holds. Also, hypothesis $\left(H_{2}\right)$ is satisfied with $L_{1}=\frac{1}{10 e}$ and $L_{2}=\frac{1}{36}$. Via some straightforward computations, we see that $A_{1}=1$, 
$A_{2}=\frac{1}{2}, \Lambda_{f_{1}}=\frac{2}{5 e \sqrt{\pi}}<1, \Lambda_{f_{2}}=\frac{1}{9 \sqrt{\pi}}<1, \Delta_{f_{1}}=1+\frac{4}{\sqrt{\pi}}$, and $\Delta_{f_{2}}=2+\frac{2}{\sqrt{\pi}}$. Thus all the suppositions in Theorem 4 are satisfied. An application of Theorem 4 shows that problem (43) has a unique solution in $C[0,1]$. Moreover, since $\Delta=1-\mathcal{C}_{\theta_{1}} \mathcal{C}_{\theta_{1}}=1-\frac{1}{90 e \pi} \neq 0$, where $\mathcal{C}_{\theta_{1}}=\frac{1}{5 e \sqrt{\pi}}$ and $\mathcal{C}_{\theta_{2}}=\frac{1}{18 \sqrt{\pi}}$, Theorem 6 shows that coupled system (43) is U-H and G-U-H stable.

Example 3 Consider the special case when $\psi(t)=\log t$ of the coupled system of TVP for Hilfer-Hadamard FDE

$$
\left\{\begin{array}{l}
D_{1^{+}}^{\frac{1}{4}, \frac{1}{3} ; \log t} y(t)=\frac{1}{5+2^{t}} \frac{x^{2}(t)+|x(t)|}{1+|x(t)|}+\sqrt{2}, \quad t \in(1, e], \\
D_{1^{+}}^{\frac{1}{4}, \frac{1}{3} ; \log t} x(t)=\frac{e^{-\log t}}{e^{\log t}+8} \frac{|y(t)|}{1+|y(t)|}+\frac{3}{2}, \quad t \in(1, e], \\
y(e)=1, \quad x(e)=2 .
\end{array}\right.
$$

Through comparing coupled system (3)-(4) with (44), we have $\theta_{1}=\theta_{2}=\frac{1}{4}, \eta_{1}=\eta_{2}=\frac{1}{3}$, $\varsigma_{1}=\varsigma_{2}=\frac{1}{2}, \psi(t)=\log t, a=1, T=e, w_{1}=1, w_{2}=2$ and $f_{1}(t, u)=\frac{1}{5+2^{t}} \frac{u^{2}+u}{1+u}+\sqrt{2}$ and $f_{2}(t, v)=$ $\frac{e^{-\log t}}{e^{\log t}+8} \frac{v}{1+v}+\frac{3}{2}$ for $u, v \in \mathbb{R}, t \in(1, e]$. Evidently, the functions $f_{1}, f_{2} \in C_{\frac{1}{2} ; \log }[1, e]$. So, condition $\left(H_{1}\right)$ holds. It is simple to verify that

$$
\begin{aligned}
& \left|f_{1}(t, u)-f_{1}\left(t, u^{*}\right)\right| \leq \frac{1}{7}\left|u-u^{*}\right|, \quad t \in(1, e], u, u^{*} \in \mathbb{R}, \\
& \left|f_{2}(t, v)-f_{2}\left(t, v^{*}\right)\right| \leq \frac{1}{9}\left|v-v^{*}\right|, \quad t \in(1, e], v, v^{*} \in \mathbb{R} .
\end{aligned}
$$

Thus, hypothesis $\left(H_{2}\right)$ is satisfied with $L_{1}=\frac{1}{7}$ and $L_{2}=\frac{1}{9}$. Through some easy calculations, we infer that $A_{1}=\sqrt{2}, A_{2}=\frac{3}{2}, \Lambda_{f_{1}} \approx 0.4<1, \Lambda_{f_{2}} \approx 0.3<1, \Delta_{f_{1}} \approx 4.1$, and $\Delta_{f_{2}} \approx 5.3$. Since every supposition in Theorem 4 is satisfied, problem (44) has a unique solution in $C_{\frac{1}{2}, \log }^{\frac{1}{2}}[1, e]$. Further, as shown in Theorem 6, for every $\epsilon=\max \left(\epsilon_{1}, \epsilon_{2}\right)>0$, if $(\widetilde{y}, \tilde{x}) \in E \times E$ satisfies

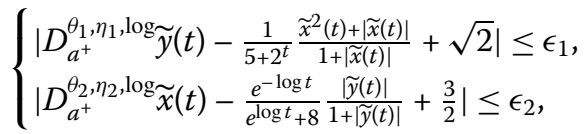

there exists a unique solution $(y, x) \in E \times E$ such that

$$
\|(\widetilde{y}, \widetilde{x})-(y, x)\|_{E \times E} \leq \lambda \epsilon
$$

where

$$
\lambda=\left(\frac{\overline{\mathcal{A}_{1}}+\mathcal{C}_{\theta_{2}} \overline{\mathcal{A}_{1}}+\overline{\mathcal{A}_{2}}+\mathcal{C}_{\theta_{1}} \overline{\mathcal{A}_{2}}}{\Delta}\right) \approx 5.4>0,
$$

$\overline{\mathcal{A}_{1}}=\overline{\mathcal{A}_{2}}=\frac{2}{\Gamma\left(\frac{5}{4}\right)}, \mathcal{C}_{\theta_{1}}=\frac{\sqrt{\pi}}{7 \Gamma\left(\frac{3}{4}\right)}, \mathcal{C}_{\theta_{2}}=\frac{\sqrt{\pi}}{9 \Gamma\left(\frac{3}{4}\right)}$, and $\Delta=1-\mathcal{C}_{\theta_{1}} \mathcal{C}_{\theta_{1}}=1-\frac{\pi}{63 \Gamma\left(\frac{3}{4}\right)^{2}} \neq 0$.

Hence coupled system (44) is U-H and G-U-H stable. Some graphical presentation of Example 3 is shown in Fig. 1. 

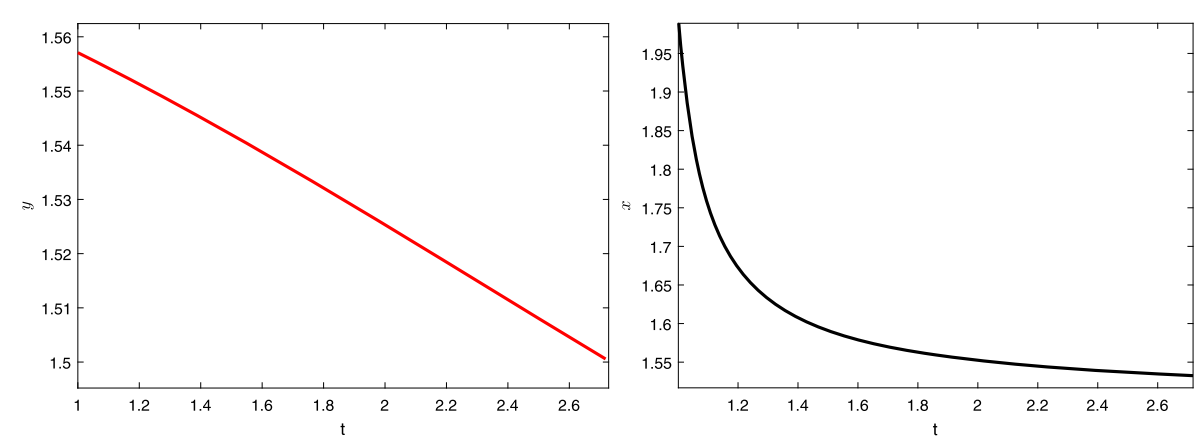

Figure 1 Graphical representation of approximate solution for Example 3 at various fractional order of coupled system (44)

\section{Acknowledgements}

We are thankful to the reviewers for their careful reading and constructive suggestions which improved this paper very much.

\section{Funding}

Funding source is not available.

\section{Abbreviations}

FDEs, Fractional differential equations; TVPs, Terminal value problems; RL, Riemann-Liouville; U-H, Ulam-Hyers; G-U-H, Generalized Ulam-Hyers.

\section{Availability of data and materials}

This is not applicable in our paper.

\section{Competing interests}

None of the authors has conflict of interests regarding this work.

\section{Authors' contributions}

All authors contributed to this manuscript and approved the final version.

\section{Author details}

'Department of Mathematics, Dr. Babasaheb Ambedkar Marathwada University, Aurangabad, India. ${ }^{2}$ Department of Mathematics, Hodeidah University, AL-Hodeidah, Yemen. ${ }^{3}$ Department of Mathematics, University of Malakand, Chakdara, 18000, Dir(L), Khyber Pakhtunkhwa, Pakistan.

\section{Publisher's Note}

Springer Nature remains neutral with regard to jurisdictional claims in published maps and institutional affiliations.

Received: 14 April 2020 Accepted: 15 June 2020 Published online: 25 June 2020

\section{References}

1. Abdo, M.S., Ibrahim, A.G., Panchal, S.K.: Nonlinear implicit fractional differential equation involving $\psi$-Caputo fractional derivative. Proc. Jangjeon Math. Soc. 22(3), 387-400 (2019)

2. Abdo, M.S., Panchal, S.K.: Fractional integro-differential equations involving $\psi$-Hilfer fractional derivative. Adv. Appl. Math. Mech. 11(2), 338-359 (2019)

3. Abdo, M.S., Panchal, S.K., Hussien, H.S.: Fractional integro-differential equations with nonlocal conditions and $\psi$-Hilfer fractional derivative. Math. Model. Anal. 24(4), 564-584 (2019)

4. Abdo, M.S., Panchal, S.K., Saeed, A.M.: Fractional boundary value problem with $\psi$-Caputo fractional derivative. Proc. Indian Acad. Sci. Math. Sci. 129(5), 65 (2019)

5. Aftabizadeh, A.R., Lakshmikantham, V.: On the theory of terminal value problems for ordinary differential equations. Nonlinear Anal., Theory Methods Appl. 5(11), 1173-1180 (1981)

6. Ali, Z., Kumam, P., Shah, K., Zada, A.: Investigation of Ulam stability results of a coupled system of nonlinear implicit fractional differential equations. Mathematics 7(4), 341 (2019)

7. Ali, Z., Zada, A., Shah, K.: Ulam stability to a toppled systems of nonlinear implicit fractional order boundary value problem. Bound. Value Probl. 2018, 175, 1-16 (2018)

8. Ali, Z., Zada, A., Shah, K.: On Ulam's stability for a coupled systems of nonlinear implicit fractional differential equations. Bull. Malays. Math. Sci. Soc. 42(5), 2681-2699 (2019)

9. Almalahi, M.A., Abdo, M.S., Panchal, S.K.: Existence and Ulam-Hyers-Mittag-Leffler stability results of $\psi$-Hilfer nonlocal Cauchy problem. Rend. Circ. Mat. Palermo, II. Ser (2020). https://doi.org/10.1007/s12215-020-00484-8

10. Almeida, R.: A Caputo fractional derivative of a function with respect to another function. Commun. Nonlinear Sci. Numer. Simul. 44, 460-481 (2017) 
11. Almeida, R.: Fractional differential equations with mixed boundary conditions. Bull. Malays. Math. Sci. Soc. 42(4), 1687-1697 (2019)

12. Atanackovic, T.M., Pilipovic, S., Stankovic, B., Zorica, D.: Fractional Calculus with Applications in Mechanics: Wave Propagation, Impact and Variational Principles. Wiley, New York (2014)

13. Benchohra, M., Bouriah, S., Nieto, J.J.: Terminal value problem for differential equations with Hilfer-Katugampola fractional derivative. Symmetry 11(5), 672 (2019)

14. Ford, N.J., Morgado, M.L., Rebelo, M.: A nonpolynomial collocation method for fractional terminal value problems. J. Comput. Appl. Math. 275, 392-402 (2015)

15. Hallam, T.G.: A comparison principle for terminal value problems in ordinary differential equations. Trans. Am. Math. Soc. 169, 49-57 (1972)

16. Jarad, F., Abdeljawad, T., Baleanu, D.: Caputo-type modification of the Hadamard fractional derivatives. Adv. Differ. Equ. 2012(1), 142 (2012)

17. Kassim, M.D., Tatar, N.E.: Well-posedness and stability for a differential problem with Hilfer-Hadamard fractional derivative. Abstr. Appl. Anal. 2013, 605029 (2013)

18. Katugampola, U.N.: A new approach to generalized fractional derivatives. Bull. Math. Anal. Appl. 6(4), 1-15 (2014)

19. Kilbas, A.A., Srivastava, H.M., Trujillo, J.J.: Theory and Applications of Fractional Differential Equations, vol. 204. Elsevier, Amsterdam (2006)

20. Magin, R.L.: Fractional Calculus in Bioengineering. Begell House Inc. Publisher (2006)

21. Magin, R.L.: Fractional calculus models of complex dynamics in biological tissues. Comput. Math. Appl. 59, 1586-1593 (2010)

22. Mali, A.D., Kucche, K.D.: Nonlocal boundary value problem for generalized hilfer implicit fractional differential equations (2020) arXiv preprint. arXiv:2001.08479

23. Manam, S.R.: Multiple integral equations arising in the theory of water waves. Appl. Math. Lett. 24, 1369-1373 (2011)

24. Oliveira, D.S., de Oliveira, E.C.: Hilfer-Katugampola fractional derivatives. Comput. Appl. Math. 37(3), 3672-3690 (2018)

25. Seemab, A., Rehman, M.U., Alzabut, J., Hamdi, A.: On the existence of positive solutions for generalized fractional boundary value problems. Bound. Value Probl. 2019(1), 186 (2019)

26. Shah, K., Wang, J., Khalil, H., Khan, R.A.: Existence and numerical solutions of a coupled system of integral BVP for fractional differential equations. Adv. Differ. Equ. 2018(1), 149 (2018)

27. Shah, S.H., Rehman, M.: A note on terminal value problems for fractional differential equations on infinite interval. Appl. Math. Lett. 52, 118-125 (2016)

28. Shreve, W.E.: Terminal value problems for second order nonlinear differential equations. SIAM J. Appl. Math. 18 783-791 (1970)

29. Sousa, J.V.D.C., de Oliveira, E.C.: On the $\psi$-Hilfer fractional derivative. Commun. Nonlinear Sci. Numer. Simul. 60, $72-91$ (2018)

30. Sousa, J.V.D.C., de Oliveira, E.C.: Ulam-Hyers stability of a nonlinear fractional Volterra integro-differential equation Appl. Math. Lett. 81, 50-56 (2018)

31. Sousa, J.V.D.C., De Oliveira, E.C.: On the Ulam-Hyers-Rassias stability for nonlinear fractional differential equations using the $\psi$-Hilfer operator. J. Fixed Point Theory Appl. 20(3), 96 (2018)

32. Sousa, J.V.D.C., de Oliveira, E.C.: A Gronwall inequality and the Cauchy-type problem by means of $\psi$-Hilfer operator. Differ. Equ. Appl. 11(1), 87-106 (2019)

33. Sousa, J.V.D.C., De Oliveira, E.C.: Leibniz type rule: $\psi$-Hilfer fractional operator. Commun. Nonlinear Sci. Numer. Simul. 77, 305-311 (2019)

34. Sousa, J.V.D.C., Rodrigues, F.G., De Oliveira, E.C.: Stability of the fractional Volterra integro-differential equation by means of $\psi$-Hilfer operator. Math. Methods Appl. Sci. 42(9), 3033-3043 (2019)

35. Zaky, M.A.: Recovery of high order accuracy in Jacobi spectral collocation methods for fractional terminal value problems with non-smooth solutions. J. Comput. Appl. Math. 357, 103-122 (2019)

36. Zhou, Y.: Basic Theory of Fractional Differential Equations, vol. 6. World Scientific, Singapore (2014)

\section{Submit your manuscript to a SpringerOpen ${ }^{\circ}$ journal and benefit from:}

- Convenient online submission

- Rigorous peer review

- Open access: articles freely available online

- High visibility within the field

- Retaining the copyright to your article

Submit your next manuscript at $\boldsymbol{~ s p r i n g e r o p e n . c o m ~}$ 